\section{Check for updates}

Cite this: J. Mater. Chem. C, 2017, 5, 11927

Received 30th August 2017, Accepted 15th October 2017

DOI: $10.1039 / \mathrm{ctc03959j}$

rsc.li/materials-c

\title{
Novel 4,8-benzobisthiazole copolymers and their field-effect transistor and photovoltaic applications $\dagger$
}

\author{
Gary Conboy, ${ }^{a}$ Rupert G. D. Taylor, (D) ${ }^{a}$ Neil J. Findlay, ${ }^{a}$ Alexander L. Kanibolotsky, ${ }^{\text {ab }}$ \\ Anto R. Inigo, ${ }^{2}$ Sanjay S. Ghosh, ${ }^{C}$ Bernd Ebenhoch, ${ }^{C}$ Lethy Krishnan Jagadamma, (D) \\ Gopala Krishna V. V. Thalluri, ${ }^{C}$ Muhammad T. Sajjad, (D) ${ }^{c}$ Ifor D. W. Samuel (D) ${ }^{c}$ and \\ Peter J. Skabara (iD *a
}

\begin{abstract}
A series of copolymers containing the benzo[1,2-d:4,5- $\left.d^{\prime}\right]$ bis(thiazole) (BBT) unit has been designed and synthesised with bisthienyl-diketopyrrolopyrrole (DPP), dithienopyrrole (DTP), benzothiadiazole (BT), benzodithiophene (BDT) or 4,4'-dialkoxybithiazole (BTz) comonomers. The resulting polymers possess a conjugation pathway that is orthogonal to the more usual substitution pathway through the 2,6-positions of the BBT unit, facilitating intramolecular non-covalent interactions between strategically placed heteroatoms of neighbouring monomer units. Such interactions enable a control over the degree of planarity through altering their number and strength, in turn allowing for tuning of the band gap. The resulting 4,8-BBT materials gave enhanced mobility in p-type organic field-effect transistors of up to $2.16 \times 10^{-2} \mathrm{~cm}^{2} \mathrm{~V}^{-1} \mathrm{~s}^{-1}$ for PDPP2ThBBT and good solar cell performance of up to $4.45 \%$ power

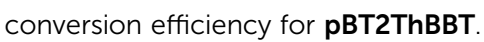

\section{Introduction}

The use of organic materials as the active components of electronic devices has been an area of much research over the past few decades. Such interest over inorganic materials (e.g. silicon based), is largely due to their inherent tunability, solubility and flexibility, allowing for devices to be made from organic materials with finely tuned properties that can be processed from solution onto flexible substrates..$^{1-3}$ The development of new materials for use in organic field-effect transistors (OFETs) is facilitating great advancements in charge carrier mobility, with these materials finding many applications in modern technology such as biosensors, ${ }^{4}$ addressing in active matrix light-emitting diodes (AMOLEDs) ${ }^{5}$ and as a low cost, flexible alternative to amorphous silicon in radiofrequency identification tagging (RFIDs). ${ }^{6}$ In order for organic materials to find useful applications, the mobility should be at least comparable to amorphous silicon, which has a hole mobility $(\mu)$ of $0.1 \leq \mu \leq 1 \mathrm{~cm}^{2} \mathrm{~V}^{-1} \mathrm{~s}^{-1}$. There are now many reports of

\footnotetext{
${ }^{a}$ WestCHEM, Department of Pure and Applied Chemistry, Thomas Graham Building, University of Strathclyde, Glasgow, G1 1XL, UK. E-mail: peter.skabara@glasgow.ac.uk

${ }^{b}$ Institute of Physical-Organic Chemistry and Coal Chemistry, 02160 Kyiv, Ukraine 'Organic Semiconductor Centre, SUPA, School of Physics and Astronomy, University of St. Andrews, North Haugh, St. Andrews, KY16 9SS, UK

$\dagger$ Electronic supplementary information (ESI) available. See DOI: 10.1039/c7tc03959j
}

organic materials that have values $\mu>10 \mathrm{~cm}^{2} \mathrm{~V}^{-1} \mathrm{~s}^{-1} \cdot{ }^{8-15}$ Many of these leading candidates currently require pre-treatment of the $\mathrm{SiO}_{2}$ substrate to control polymer chain self-assembly ${ }^{12}$ or increase wettability, ${ }^{2}$ and/or thermal annealing at high temperature ${ }^{16}$ in order to achieve a suitable morphology capable of affording high mobility. ${ }^{17}$ Such processes add to the time, cost and complexity of fabrication and, as such, materials which show high mobility with minimal processing steps are highly desired for industrial use.

Organic semiconductors are also often studied for use as functional active layer materials in organic photovoltaic (OPV) devices, as they can be engineered to have a broad absorption across the solar spectrum. When combined with a suitable acceptor species, resultant organic solar cells can have power conversion efficiencies (PCEs) of over $12 \%{ }^{18,19}$ In order to minimise the band gap, as well as to increase the carrier mobility and self-assembly within the bulk phase, it is beneficial to place alternating conjugated donor and acceptor units into the backbone of the conductive material to facilitate a push-pull effect. ${ }^{20,21}$ Such a structure is readily achievable in polymeric form through the copolymerisation of suitably functionalised donor and acceptor units. Many of these units contain multiple heteroatoms that offer further advantages, such as planarisation and strong interchain packing due to a combination of $\pi-\pi$ stacking and non-covalent heteroatom/weak hydrogen bonding interactions in the bulk material. ${ }^{2-25}$ For example, the use of 

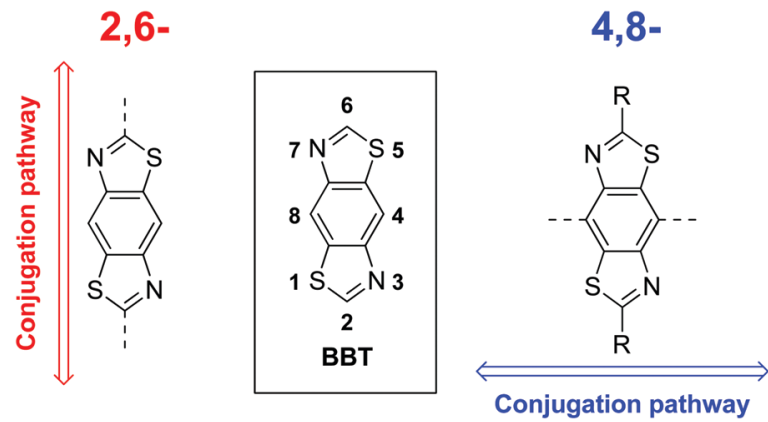

Fig. 1 Typical 2,6-BBT conjugation pathway (left) and alternate 4,8-BBT conjugation pathway (right).

thiazole (rather than thiophene) facilitates these advantages in combination with deeper highest occupied molecular orbital (HOMO) and lowest unoccupied molecular orbital (LUMO) energy levels ${ }^{26}$ to give increased device performance in both OFETs $^{27,28}$ and OPVs. ${ }^{29,30}$ Additionally, intermolecular noncovalent interactions in the bulk are evident, further contributing to improved charge carrier properties. ${ }^{26,31-33}$

Recently we highlighted the use of a thiazole-containing benzobisthiazole (BBT) unit with an orthogonal (4,8- vs. traditional 2,6-) conjugation pathway (Fig. 1). ${ }^{25}$ Whilst BBT units possessing the 2,6-conjugation pathway are common and well-studied, those with 4,8-substitution have been underexplored in comparison, ${ }^{25,34-38}$ despite offering a better template with which to facilitate planarising intramolecular non-covalent interactions with neighbouring heterocycles. ${ }^{25}$ By carefully selecting the type and location of heteroatoms in the flanking heterocycles (Fig. 2), the planarity of the resultant molecule or polymer can be tuned and, in turn, their solubility and energy gap can be modified.

Non-covalent interactions are defined as contact distances between two atoms (often heteroatoms) which are shorter than the sum of the van der Waals radii of the two corresponding atoms. Through a combination of X-ray crystallography and computational simulations we have previously demonstrated that the inclusion of thiophene units either side of the BBT heterocycle (Fig. 2: $\mathrm{X}=\mathrm{CH}, \mathrm{Y}=\mathrm{S}$ ) gives a twisted, non-rigid structure. In the orientation depicted in Fig. $2, \mathrm{C}-\mathrm{H} \cdots \mathrm{N}$ hydrogen bonding interactions are offset by the repulsive $\mathrm{S} \cdots \mathrm{S}$ interactions, whilst $\mathrm{S} \cdots \mathrm{N}$ interactions (when the flanking heterocycles are flipped $180^{\circ}$ relative to Fig. 2) are deterred by steric hindrance between the $\mathrm{C}-\mathrm{H}$ and $\mathrm{S}$ groups. ${ }^{25}$ Conversely, utilising thiazole moieties in place of thiophene (Fig. 2: $\mathrm{X}=\mathrm{S}, \mathrm{Y}=\mathrm{N}$ ) results in four

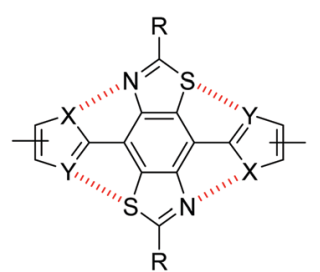

Fig. 2 Non-covalent intramolecular interactions between $\mathrm{S}$ and $\mathrm{N}$ atoms of a BBT core and flanking heterocycles. intramolecular $\mathrm{S} \cdots \mathrm{N}$ non-covalent interactions and a highly planarised structure (maximum torsion angle of $5.1^{\circ}$ across the $\mathrm{C}-\mathrm{C}$ bond connecting the BBT unit and heterocycle), and the use of furan (Fig. 2: $\mathrm{X}=\mathrm{CH}, \mathrm{Y}=\mathrm{O}$ ) leads to a similarly highly planarised structure (maximum torsion angle of $4.1^{\circ}$ ) through non-covalent $\mathrm{S} \cdots \mathrm{O}$ interactions. ${ }^{25}$ These, and other results, have shown that non-covalent $\mathrm{S} \cdots \mathrm{N}$ and $\mathrm{S} \cdots \mathrm{O}$ contacts offer favourable interactions of comparable strength, whilst $\mathrm{S} \cdots \mathrm{S}$ interactions are repulsive and $\mathrm{N} \cdots \mathrm{O}$ interactions are weak/negligible. It is important to note that additional intramolecular hydrogen bonding interactions with the BBT nitrogen atoms may be playing a role when $\mathrm{X}=\mathrm{CH}$ (Fig. 2), however it has been unequivocally shown that heteroatom-heteroatom interactions are influential on the structure of such molecules. ${ }^{25}$

In this work we describe the synthesis of a series of BBT copolymers conjugated along the 4,8-substitution pathway (Fig. 1) and report their semiconducting properties in OFET and OPV devices. Bisthienyl-diketopyrrolopyrrole (DPP), dithienopyrrole (DTP), benzothiadiazole (BT), benzodithiophene (BDT) and 4,4'dialkoxybithiazole (BTz) were selected as comonomers due to their reported behaviour in high performance OFET $^{32,39}$ and $\mathrm{OPV}^{40}$ devices, but also to allow for band gap variation through HOMO and LUMO energy level tuning and to provide varying degrees of planarity through non-covalent heteroatom interactions (as discussed above).

\section{Synthesis}

The targeted BBT-containing polymers were realised via Stille or Suzuki cross-coupling mediated polymerisations between alkylated 4,8-dibromo-BBT and suitably functionalised bisthienyldiketopyrrolopyrrole (DPP), ${ }^{41,42}$ dithienopyrrole (DTP), benzothiadiazole (BT) ${ }^{43}$ benzodithiophene (BDT) ${ }^{44}$ or $4,4^{\prime}$-dialkoxybithiazole $(\mathrm{BTz})^{32}$ monomers. Each polymer was then end-capped with thiophene units via subsequent Stille or Suzuki cross-coupling reactions with commercial tributyl(thiophen-2-yl)stannane (or thiophen2-ylboronic acid in the case of pDPPThBBT) then 2-bromothiophene. Polymers containing a biheterocyclic bridge between donor/acceptor units were prepared as random copolymers using 2,5-bis(trimethylstannyl)thiophene ${ }^{45}$ (pDPP2ThBBT and pBT2ThBBT) or 2,5-bis(trimethylstannyl)furan ${ }^{45}$ (pDPPThFBBT) as a third monomer. As a result, their structures shown in Scheme 1 represent an idealised structure in each case. Detailed synthetic procedures for all monomers and polymers can be found in the ESI. $\dagger$

Molecular weights (Table 1) of the resultant polymers were determined by gel permeation chromatography (GPC) in chloroform or $o$-dichlorobenzene solution and show a range of different values (14.4-96.0 $\left.\mathrm{kg} \mathrm{mol}^{-1}\right)$. The limiting factor in molecular weight for each polymer is solubility - all polymers precipitated from solution during their respective polymerisations. Despite their very different molecular weights, pDPP2ThBBT and pDPPThFBBT have very similar solubility, suggesting that there is reduced rotational freedom in pDPPThFBBT due to intramolecular $\mathrm{S} \cdots \mathrm{O}$ and $\mathrm{C}-\mathrm{H} \cdots \mathrm{N}$ interactions, ${ }^{25}$ facilitated by the 


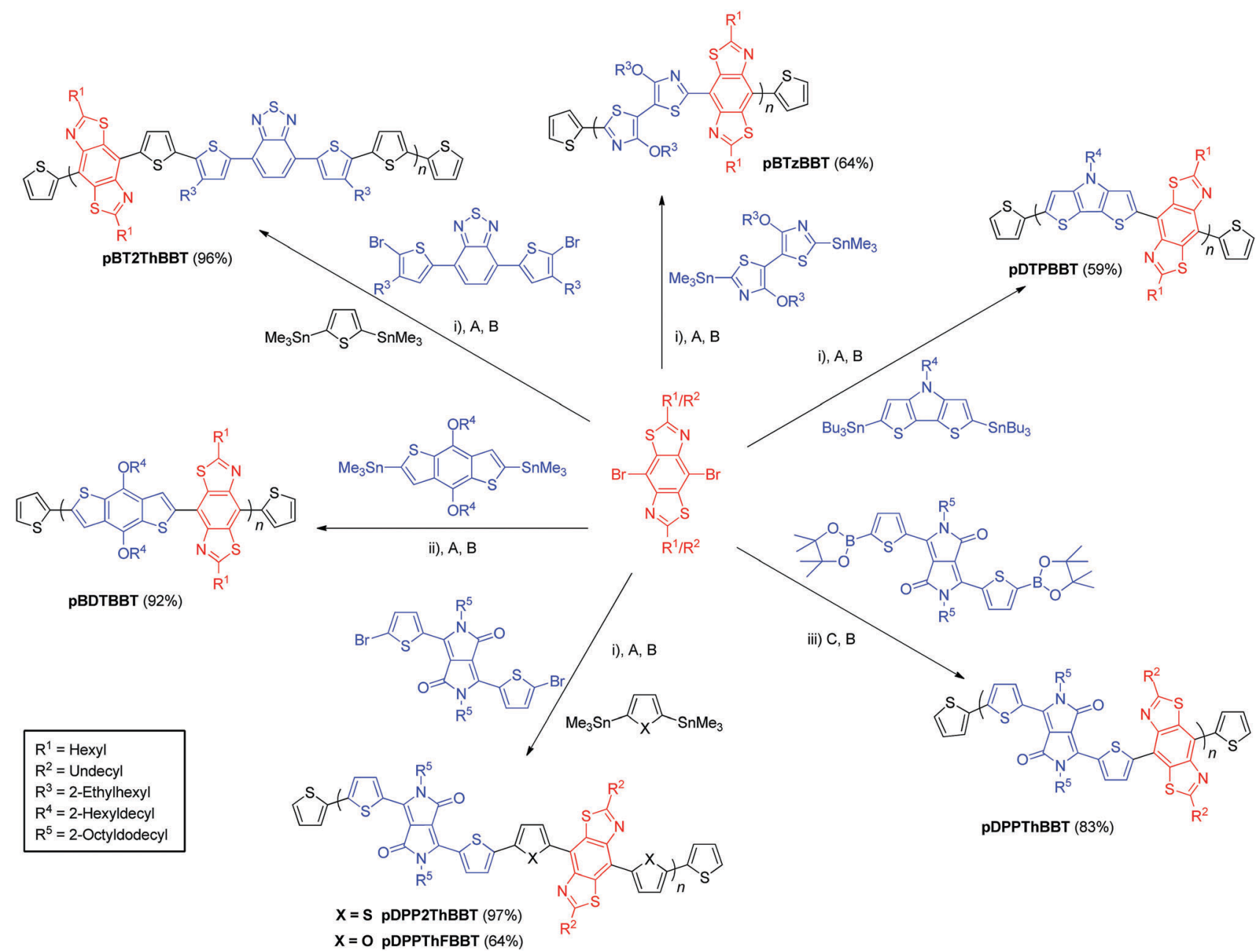

Scheme 1 Synthesis of BBT-containing copolymers. Yields calculated from repeat units as drawn. Reagents and conditions: (i) $\mathrm{Pd}_{2}(\mathrm{dba})_{3}, \mathrm{P}\left(0-\mathrm{tol}_{3}\right.$, chlorobenzene, $160{ }^{\circ} \mathrm{C}, \mu \mathrm{W}$. (ii) $\mathrm{Pd}_{2}(\mathrm{dba})_{3}, \mathrm{P}(\mathrm{o} \text {-tol })_{3}$, toluene, reflux. (iii) $\mathrm{Pd}_{2}(\mathrm{dba})_{3}, \mathrm{P}(\mathrm{o} \text {-tol })_{3}, \mathrm{~K}_{3} \mathrm{PO}_{4}, \mathrm{THF}$, reflux. End-capping reagents: (A) tributyl(thiophen-2-yl)stannane. (B) 2-Bromothiophene. (C) Thiophen-2-ylboronic acid.

Table 1 GPC, optical, electrochemical and thermal decomposition $\left(T_{d}\right)$ data for the 4,8-BBT copolymers. GPC data not obtained for pBTzBBT and pDTPBBT due to incomplete polymer solubility

\begin{tabular}{|c|c|c|c|c|c|c|c|c|c|c|c|}
\hline Polymer & $M_{\mathrm{w}}\left(\mathrm{kg} \mathrm{mol}{ }^{-1}\right)$ & PDI & $E_{\mathrm{g}}^{\mathrm{opt} c}(\mathrm{eV})$ & $E_{\mathrm{g}}^{\text {elect } d}(\mathrm{eV})$ & \multicolumn{2}{|l|}{ Solution $^{e}$} & \multicolumn{2}{|l|}{ Film } & $\operatorname{HOMO}^{d}(\mathrm{eV})$ & $\mathrm{LUMO}^{d}(\mathrm{eV})$ & $T_{\mathrm{d}}\left({ }^{\circ} \mathrm{C}\right)$ \\
\hline pBTzBBT & - & - & 1.53 & 1.69 & $685,740^{f}$ & 795 & $657,731^{f}$ & 808 & -4.69 & -3.00 & 360 \\
\hline pDPP2ThBBT & $96.0^{a}$ & 2.75 & 1.39 & 1.43 & 765 & 865 & 748 & 895 & -5.10 & -3.67 & 411 \\
\hline pDPPThFBBT & $18.0^{a}$ & 2.04 & 1.43 & 1.26 & $716^{f}, 751$ & 855 & 705,756 & 870 & -4.86 & -3.60 & 365 \\
\hline pBT2ThBBT & $17^{a}$ & 1.80 & 1.58 & - & 548 & 690 & 620 & 785 & -5.20 & - & 451 \\
\hline
\end{tabular}

${ }^{a}$ Calculated from GPC using $0.5 \mathrm{mg} \mathrm{ml}^{-1}$ solutions in chlorobenzene at $80{ }^{\circ} \mathrm{C} .{ }^{b}$ Calculated from GPC using $1 \mathrm{mg} \mathrm{ml}^{-1} \mathrm{solutions}$ in chloroform at $22{ }^{\circ} \mathrm{C}$. ${ }^{c}$ Calculated from the onset of the longest solid state wavelength absorption peak. ${ }^{d}$ Found from CV, using the onset of redox activity and referenced to $\mathrm{Fc} / \mathrm{Fc}^{+}(-4.8 \mathrm{eV}) .{ }^{e}$ Absorption spectra obtained from $o$-dichlorobenzene solutions. ${ }^{f}$ Shoulder.

furan rings flanking the BBT unit. In contrast, the thiophene moieties flanking the BBT unit in pDPP2ThBBT result in

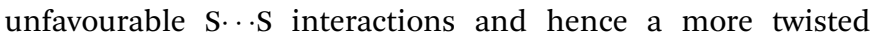
structure, allowing the growing polymer chain to remain in solution longer before precipitating. Molecular weight variation between pDPPThBBT, pBT2ThBBT and pBDTBBT (which all feature thiophene units flanking the BBT units and hence contain unfavourable $\mathrm{S} \cdots \mathrm{S}$ interactions) is likely due to a combination of different alkyl chain lengths (leading to different solubility limits of the growing polymer chains during polymerisation) and the variety of polymerisation conditions used across the series. 


\section{Optical properties}

Absorption spectra of the 4,8-BBT copolymers were obtained in $o$-dichlorobenzene solution and as thin films. The resulting spectra (Fig. 3a-f) all show broad absorption, extending up to $900 \mathrm{~nm}$ and resulting in optical band gaps in the range 1.36-2.00 eV (as calculated from the onset of the longest wavelength solid state absorption peak). All of the 4,8-BBT copolymers show a red shift in their longest wavelength absorbance onset when moving from solutions to thin films due to increased intermolecular interactions. However, this red shift is very small $(\leq 35 \mathrm{~nm}$ ) for the three DPP containing copolymers (pDDPThBBT, pDDP2ThBBT and pDPPThFBBT) and pBTzBBT, indicating that these materials possess a rigid, aggregated structure even in solution. In contrast, pBT2ThBBT, pDTPBBT and pBDTBBT show a more significant red shift (up to $95 \mathrm{~nm}$ ) in their film form, suggesting enhanced order due to aggregation and molecular packing in the solid state. This is in part due to these polymers possessing thiophene groups flanking the BBT unit, allowing for a more twisted and hence less conjugated structure in solution.
Comparison of the optical band gaps reveals that the DPP containing polymers (pDDPThBBT, pDDP2ThBBT and pDPPThFBBT) have very similar optical properties, and the smallest optical band gaps $(1.36-1.43 \mathrm{eV})$ of all the $4,8-\mathrm{BBT}$ copolymers. This suggests that the strong electron-accepting nature of the DPP unit causes it to dominate the optical properties of these copolymers, resulting in red-shifted absorption. Copolymers featuring weaker acceptor units (BTz and BT) show slightly wider optical band gaps (1.53 and $1.58 \mathrm{eV}$ for pBTzBBT and pBT2ThBBT, respectively), whilst those containing electron donating units have the widest optical band gaps (1.84 and $2.00 \mathrm{eV}$ for pDTPBBT and pBDTBBT, respectively).

The optical properties of pBT2ThBBT, pBDTBBT and pDTPBBT reveal comparable absorption profiles to their equivalent 2,6-BBT copolymers (PBBTzBT-HD, ${ }^{46}$ PBTHDDT $^{47}$ and PBTDTP,${ }^{47}$ respectively, Fig. 4) when measured in solution, with either similar or slightly hypsochromically shifted absorption onsets. As thin films, the absorption properties of pDTPBBT and its 2,6-substituted counterpart (PBTDTP) ${ }^{47}$ are also similar, and result in essentially identical optical band gaps (1.84 and $1.85 \mathrm{eV}$, respectively). However, the solid state absorption
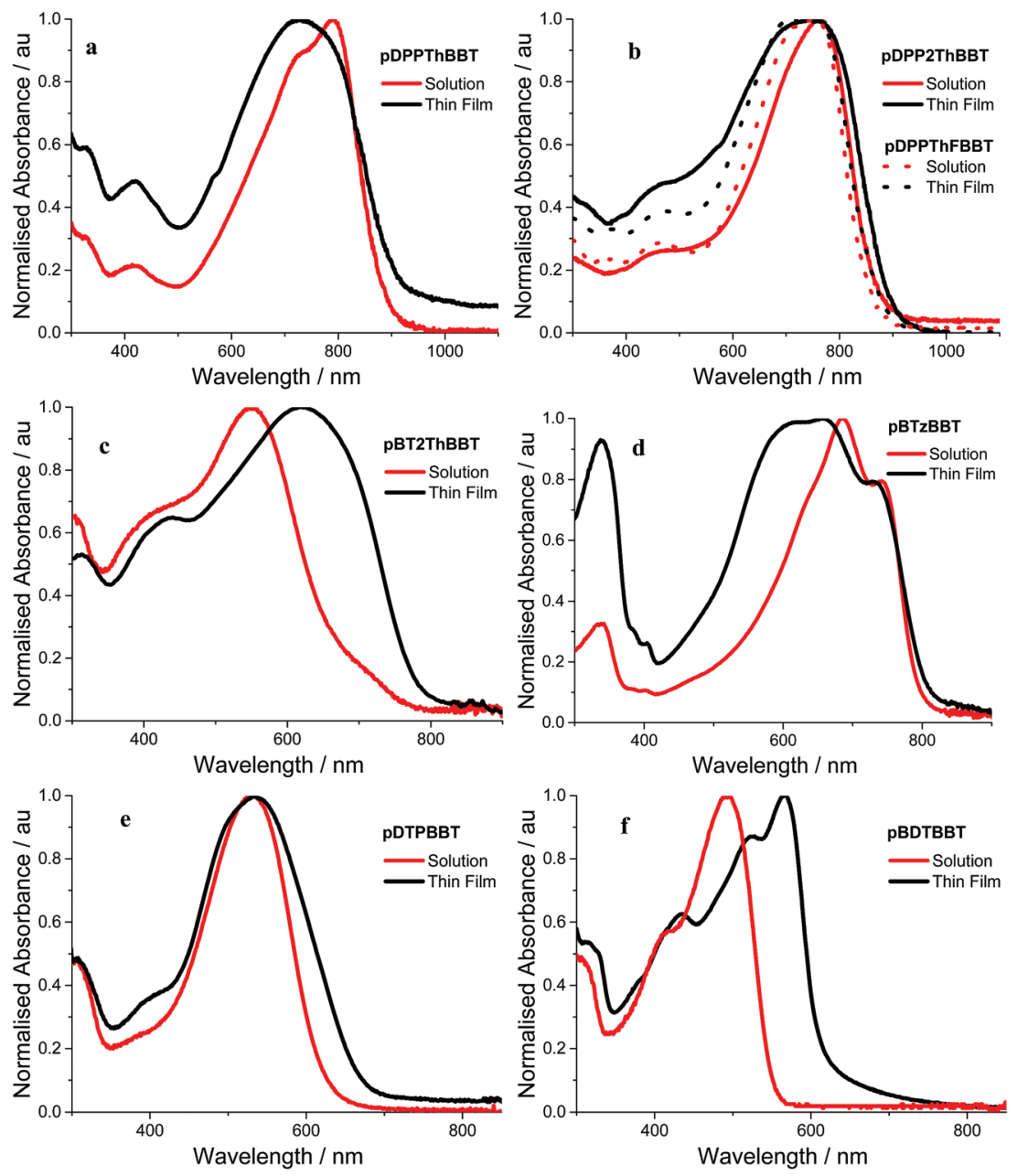

Fig. 3 Absorption spectra of (a) pDPPThBBT, (b) pDPP2ThBBT/pDPPThFBBT, (c) pBT2ThBBT, (d) pBTzBBT, (e) pDTPBBT and (f) pBDTBBT in $o$-dichlorobenzene solution (red) and as thin films drop cast from o-dichlorobenzene (a-e) or chloroform (f) (black). 

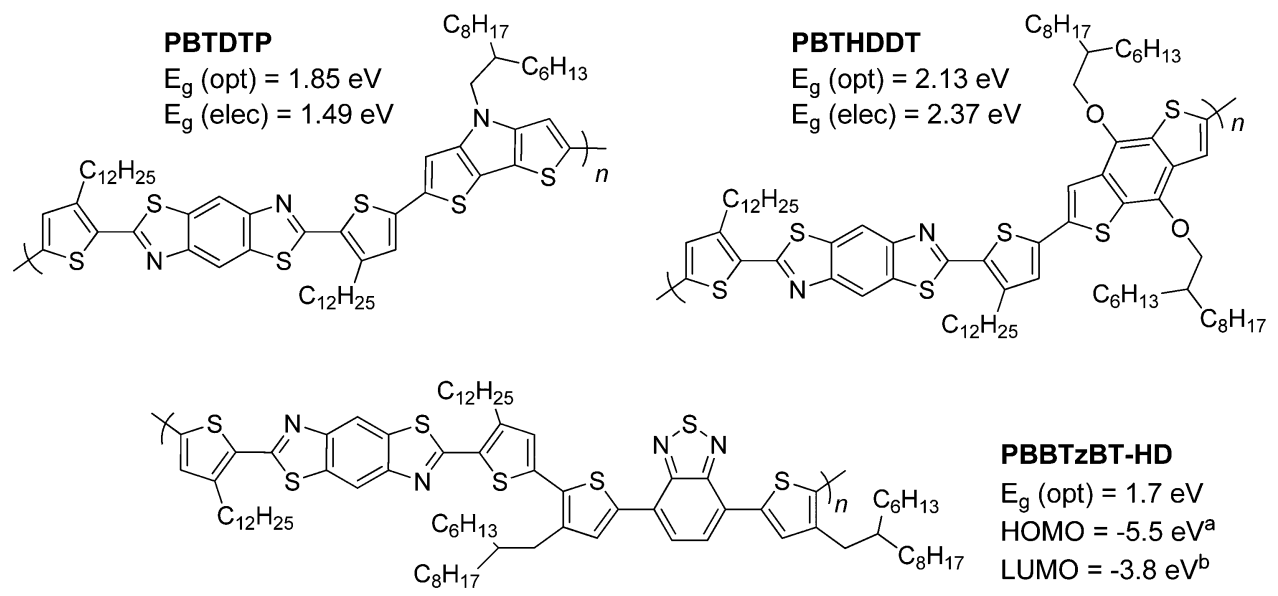

PBBTzBT-HD
$E_{g}$ (opt) $=1.7 \mathrm{eV}$
HOMO $=-5.5 \mathrm{eV}^{\mathrm{a}}$
LUMO $=-3.8 \mathrm{eV}^{\mathrm{b}}$

Fig. 4 Previously reported 2,6-BBT copolymers featuring DTP (top left), ${ }^{47}$ BDT (top right) ${ }^{47}$ and BT (bottom) units. ${ }^{46}{ }^{a}$ calculated from photoelectron yield spectroscopy, ${ }^{b}$ calculated using $\left(E_{\mathrm{g}}(\mathrm{opt})+E_{\mathrm{HO} O \mathrm{O}}\right)$.

profiles of pBT2ThBBT and pBDTBBT are bathochromically shifted compared to their 2,6-analogues, resulting in lower optical band gaps (1.58 vs. $1.7 \mathrm{eV}^{46}$ for the BT-containing polymers and 2.00 vs. $2.13 \mathrm{eV}^{47}$ for the BDT-containing polymers). Moreover, the absorption window of pBT2ThBBT is significantly broader than that of its 2,6-analogue (PBBTzBT-HD), ${ }^{46}$ allowing for increased photon absorption.

\section{Electrochemical properties}

To establish their electronic characteristics, cyclic voltammetry (CV) was performed on solutions and thin films (cast on platinum disc electrodes) of the 4,8-BBT copolymers. Electronic band gaps (Table 1) were found to be between 1.26-2.24 eV and broadly consistent with the trend in optical values. Cyclic voltammograms for all 4,8-BBT copolymers in solution and solid state are shown in Fig. S8-S20 (ESI $\dagger$ ), however pBT2ThBBT did not reveal a reduction peak at potentials as low as $-2.0 \mathrm{~V}$, preventing electrochemical determination of the polymer's LUMO level.

pDPPThBBT and pDPP2ThBBT exhibit relatively similar HOMO and LUMO energy levels, and by extension closely matching electrochemical band gaps (1.39 and $1.43 \mathrm{eV}$, respectively). pDPPThFBBT shows a slightly lower electrochemical band gap of $1.26 \mathrm{eV}$, which is likely due to a combination of the lower resonance stabilisation energy of the furan groups flanking the BBT unit and increased conjugation through planarising intramolecular $\mathrm{S}-\mathrm{O}$ interactions. The electrochemical data of the DPP-containing 4,8-BBT copolymers is consistent with the optical data in demonstrating that the strongly electron-accepting nature of the DPP unit results in it dominating the materials electronic behaviour, although thiophene or furan flanking units can fine tune this behaviour further.

In agreement with the optical data and comparing to those copolymers containing DPP, copolymers featuring weaker electron acceptor units have slightly wider electrochemical band gaps (1.69 eV for pBTzBBT), whilst those featuring electron-donating units (pDTPBBT and pBDTBBT) have much wider electrochemical band gaps (2.11 and $2.24 \mathrm{eV}$, respectively). In comparison to its 2,6-analogue, pBDTBBT has a smaller electrochemical band gap (2.24 eV vs. $2.37 \mathrm{eV}$ for PBTHDDT), ${ }^{47}$ which is in agreement to the bathochromic shift seen in the absorption measurements.

\section{Organic field-effect transistor (OFET) fabrication}

Bottom gate/bottom contact (BGBC) OFETs were prepared from commercially available (Fraunhofer Institute für Photonische Mikrosysteme IPMS, Dresden) n-doped silicon substrates with a $200 \mathrm{~nm}$ layer of thermally grown $\mathrm{SiO}_{2}$ dielectric and prefabricated interdigitated gold source/drain electrodes (channel lengths: 2.5, 5, 10 and $20 \mu \mathrm{m}$, channel width: $1 \mathrm{~cm}$ ). Unless otherwise stated, solutions were pre-stirred at $50{ }^{\circ} \mathrm{C}$ for at least three hours, then spin-coated whilst hot at $2000 \mathrm{rpm}$. Following any required annealing, the devices were dried under vacuum $\left(5 \times 10^{-2} \mathrm{mbar}\right)$, then their performance was measured on a Keithley 4200 semiconductor characterisation system, all whilst in a dry, nitrogenfilled atmosphere. Mobilities were calculated in the saturation region via the standard method using the following equation:

$$
\mu_{\mathrm{sat}}=\frac{2 L}{W C_{\mathrm{i}}} \times\left(\frac{\partial \sqrt{I_{\mathrm{ds}}}}{\partial V_{\mathrm{gs}}}\right)^{2}
$$

where $I_{\mathrm{ds}}$ is the source-drain current, $\mu$ is the carrier mobility, $V_{\mathrm{gs}}$ is the gate voltage, $L$ is the channel length, $W$ is the channel width and $C_{\mathrm{i}}$ is the capacitance per unit area of the insulator material.

Device optimisation studies were carried out for pDPP2ThBBT (selected for its solubility and relatively high molecular weight, which has been shown to result in improved charge carrier mobility $)^{48,49}$ through variation of the annealing temperature (as cast, 60, 100, 150 and $200{ }^{\circ} \mathrm{C}$ ) and solvent (chloroform, chlorobenzene or $o$-dichlorobenzene). Solutions of $10 \mathrm{mg} \mathrm{ml}^{-1}$ concentration were prepared and deposited onto the prefabricated substrates in accordance with the previously stated procedure, with the same device tested at each annealing temperature increment. To facilitate accurate comparison of the materials 
Table 2 Device characteristics for pDPP2ThBBT based OFETs, averaged across 3 devices

\begin{tabular}{|c|c|c|c|c|}
\hline Solvent & $\begin{array}{l}\text { Annealing } \\
\text { temperature }\left({ }^{\circ} \mathrm{C}\right)\end{array}$ & $\mu_{\mathrm{h}}\left(\mathrm{cm}^{2} \mathrm{~V}^{-1} \mathrm{~s}^{-1}\right)$ & $I_{\mathrm{on}} / I_{\mathrm{off}}$ & $V_{\text {th }}(\mathrm{V})$ \\
\hline \multirow[t]{5}{*}{ o-Dichlorobenzene } & As cast & $1.17 \times 10^{-2}$ & $10^{3}$ & 12 \\
\hline & 60 & $1.63 \times 10^{-2}$ & $10^{3}$ & 14 \\
\hline & 100 & $2.16 \times 10^{-2}$ & $10^{3}$ & 5 \\
\hline & 150 & $1.20 \times 10^{-2}$ & $10^{3}$ & 0 \\
\hline & 200 & $8.56 \times 10^{-3}$ & $10^{2}$ & -6 \\
\hline \multirow[t]{5}{*}{ Chlorobenzene } & As cast & $3.97 \times 10^{-3}$ & $10^{3}$ & 13 \\
\hline & 60 & $6.35 \times 10^{-3}$ & $10^{3}$ & 14 \\
\hline & 100 & $7.89 \times 10^{-3}$ & $10^{3}$ & 6 \\
\hline & 150 & $7.76 \times 10^{-3}$ & $10^{3}$ & 1 \\
\hline & $200^{a}$ & - & - & - \\
\hline \multirow[t]{5}{*}{ Chloroform } & As cast & $8.98 \times 10^{-3}$ & $10^{2}$ & 12 \\
\hline & 60 & $1.18 \times 10^{-2}$ & $10^{3}$ & 11 \\
\hline & 100 & $1.28 \times 10^{-2}$ & $10^{3}$ & 1 \\
\hline & 150 & $8.76 \times 10^{-3}$ & $10^{3}$ & -1 \\
\hline & $200^{a}$ & - & - & - \\
\hline
\end{tabular}

${ }^{a}$ Devices gave no response due to poor film morphology.

and limit further processing steps, the use of self-assembled monolayers (e.g. pentafluorobenzenethiol $)^{50}$ or processing additives were avoided. Summarised data for devices based on pDPP2ThBBT are shown in Table 2 and Fig. 5, with full device data in Fig. S21-S33 $(\mathrm{ESI} \dagger)$.

Fig. 5 shows that $o$-dichlorobenzene is the best solvent for preparing OFETs from pDPP2ThBBT, with the devices outperforming analogous devices prepared from chlorobenzene or chloroform solutions at all annealing temperatures. However, annealing was only beneficial up to $100{ }^{\circ} \mathrm{C}$, after which point further annealing resulted in a drop-off in device performance due to visibly poor film morphology. This is likely a result of the polymer exhibiting significant rigidity, meaning that modest annealing temperatures are enough to force the as cast film towards the thermodynamically most stable (crystalline) state resulting in grain boundaries. Accordingly, OFETs made from other 4,8-BBT copolymers were processed from $o$-dichlorobenzene

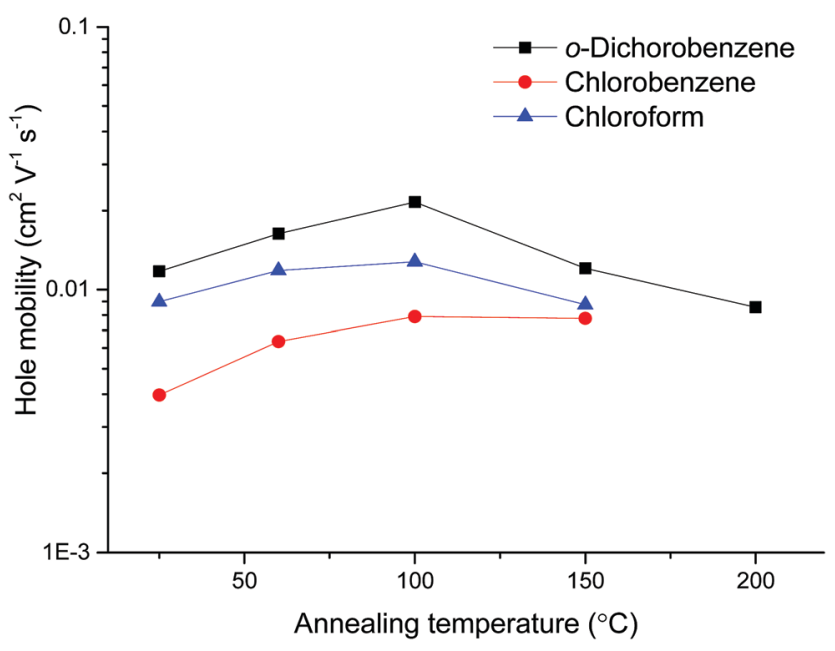

Fig. 5 Hole mobilities of OFETs based on PDPP2ThBBT as a function of annealing temperature.
Table 3 OFET device data for the 4,8-BBT copolymers. Device parameters: $B G B C$, spin coated from $10 \mathrm{mg} \mathrm{ml}^{-1} \mathrm{O}$-dichlorobenzene solution, annealed at $100{ }^{\circ} \mathrm{C}$. Averaged across 3 devices

\begin{tabular}{lllr}
\hline Polymer & $\mu_{\mathrm{h}}\left(\mathrm{cm}^{2} \mathrm{~V}^{-1} \mathrm{~s}^{-1}\right)$ & $I_{\mathrm{on}} / I_{\text {off }}$ & $V_{\text {th }}(\mathrm{V})$ \\
\hline pBTzBBT & $2.16 \times 10^{-3}$ & $10^{5}$ & -2 \\
pDPPThBBT & $3.23 \times 10^{-6}$ & $10^{5}$ & -10 \\
pDPP2ThBBT & $2.16 \times 10^{-2}$ & $10^{3}$ & 5 \\
pDPPThFBBT & $2.03 \times 10^{-3}$ & $10^{5}$ & -2 \\
pBT2ThBBT & $3.60 \times 10^{-3}$ & $10^{3}$ & -5 \\
pBDTBBT & $8.69 \times 10^{-5}$ & $10^{3}$ & -2 \\
pDTPBBT & $2.11 \times 10^{-5}$ & $10^{3}$ & 11
\end{tabular}

solution and annealed at $100{ }^{\circ} \mathrm{C}$; data obtained from these OFETs are shown in Table 3, with output and transfer characteristics in Fig. S34-S39 (ESI $\dagger$ ).

The hole mobility values obtained show a large variation, spanning four orders of magnitude. pDPP2ThBBT based OFETs gave the highest hole mobility $\left(2.16 \times 10^{-2} \mathrm{~cm}^{2} \mathrm{~V}^{-1} \mathrm{~s}^{-1}\right)$ in combination with a moderate $I_{\mathrm{on}} / I_{\text {off }}$ ratio $\left(10^{3}\right)$ and low threshold voltage $(5 \mathrm{~V})$ for a p-type device, but suffer from increased hysteresis in both output and transfer characteristics (Fig. S21-S33, ESI $\dagger$ ). This could be indicative of charge trapping in the bulk film or non-optimal device structure. pDPPThFBBT exhibits a slightly lower hole mobility $\left(2.03 \times 10^{-3} \mathrm{~cm}^{2} \mathrm{~V}^{-1} \mathrm{~s}^{-1}\right)$, possibly due to its lower molecular weight ${ }^{48,49}$ and larger threshold voltage for p-type devices $(-2 \mathrm{~V})$. This is likely due to a nonohmic contact between the gold electrode (work function -5.0 to $-5.1 \mathrm{eV})$ and the shallow HOMO of pDPPThFBBT $(-4.86 \mathrm{eV})$ resulting in poor charge injection. AFM images of the thin films of pDPP2ThBBT and pDPPThFBBT cast on OFET substrates (Fig. 6a and b) show very smooth uniform films with a root mean square (RMS) roughness of 0.55 and $0.34 \mathrm{~nm}$, respectively, and grain boundaries on the order of $0.1 \mu \mathrm{m}$ or less. Similarly acquired images of pDPPThBBT (Fig. 6c) show a much rougher film (RMS roughness of $2.82 \mathrm{~nm}$ ) with domains extending up to $5 \mu \mathrm{m}$, and grain boundaries greater than $1 \mu \mathrm{m}$ wide. This poor film morphology combined with the relatively low molecular weight of pDPPThBBT is likely the cause of the significantly inferior hole mobility $\left(3.23 \times 10^{-6} \mathrm{~cm}^{2} \mathrm{~V}^{-1} \mathrm{~s}^{-1}\right) .{ }^{49}$

To overcome the poor solubility of pBTzBBT, all solutions used for OFET fabrication were pre-stirred at $100{ }^{\circ} \mathrm{C}$ for three hours then spin-coated whilst hot to prevent precipitation of the material. In spite of this, pBTzBBT produced the most ideal OFETs of those presented in this work, with modest hole mobility $\left(2.16 \times 10^{-3} \mathrm{~cm}^{2} \mathrm{~V}^{-1} \mathrm{~s}^{-1}\right)$, but crucially a high $I_{\mathrm{on}} / I_{\mathrm{off}}$ ratio $\left(10^{5}\right)$ and a low driving voltage $(-2 \mathrm{~V})$. AFM (Fig. 6d) revealed that the film morphology of pBTzBBT was very rough (RMS roughness $=\mathbf{1 5 . 6 3} \mathrm{nm}$ ), which is likely due to the poor solubility of the polymer (despite the extra pre-treatment), meaning that further optimisation of the device preparation, or increasing the solubility of the polymer through the use of longer alkyl chains, could lead to a higher-performing solutionprocessed OFET.

An additional example of the impact of the BBT unit on OFET performance is evident in pBT2ThBBT. OFETs featuring pBT2ThBBT showed modest mobility $\left(3.60 \times 10^{-3} \mathrm{~cm}^{2} \mathrm{~V}^{-1} \mathrm{~s}^{-1}\right)$, 

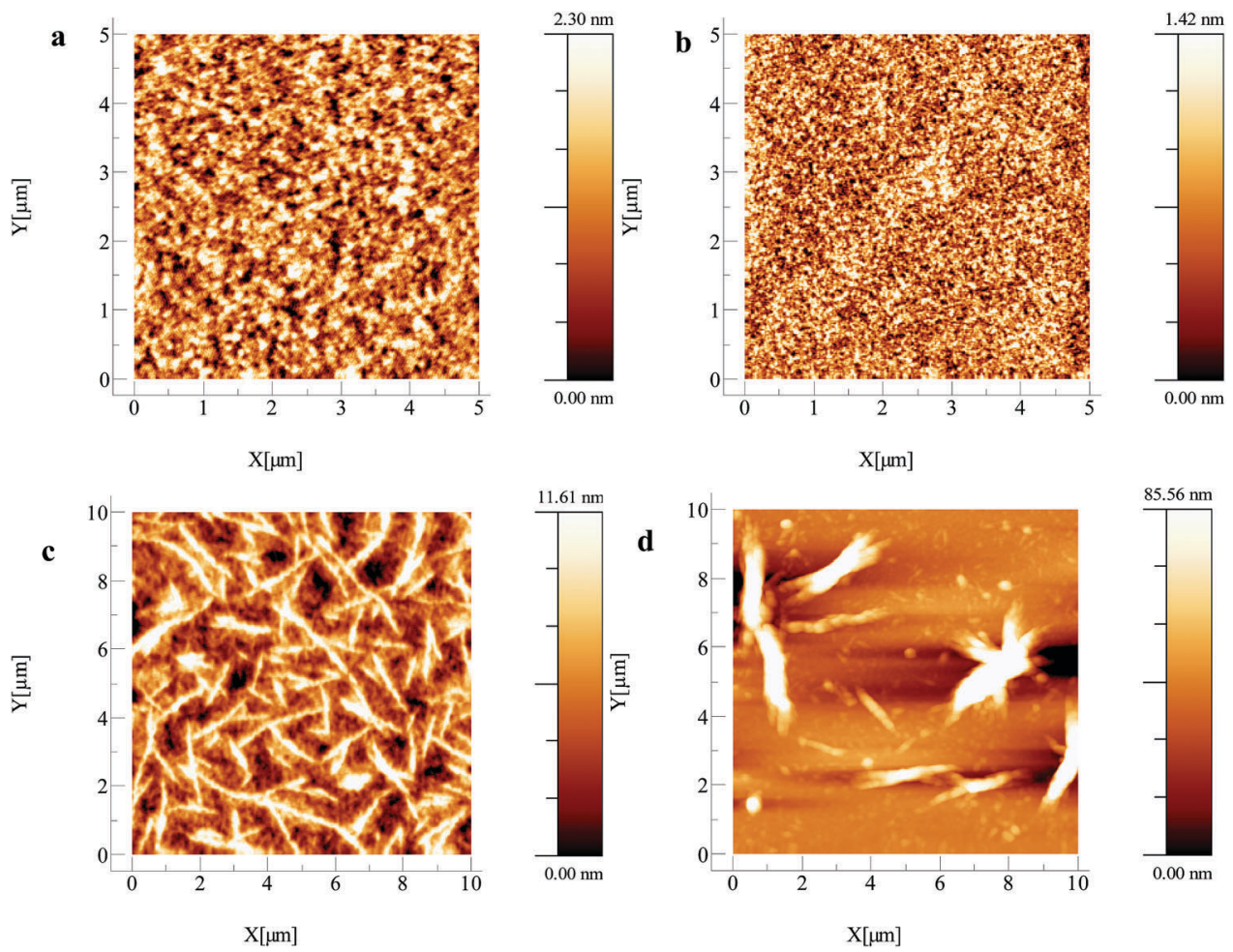

Fig. 6 AFM images of OFETs after annealing at $100{ }^{\circ} \mathrm{C}$ fabricated using (a) pDPP2ThBBT (RMS roughness $=0.55 \mathrm{~nm}$ ), (b) pDDPThFBBT $($ RMS roughness $=0.34 \mathrm{~nm})$, (c) pDPPThBBT (RMS roughness $=2.82 \mathrm{~nm}$ ) and (d) pBTzBBT (RMS roughness $=15.63 \mathrm{~nm})$.

but this is over two orders of magnitude higher than a literature example featuring only BT units, ${ }^{51}$ highlighting the enhanced crystallinity afforded by incorporation of BBT units into the polymer backbone through increased potential for non-covalent interactions and $2 \mathrm{D}$ conjugation.

\section{Organic photovoltaic (OPV) devices}

Due to the absorption characteristics in the visible spectral range, narrow band gap and efficient charge carrier transport properties of many of these 4,8-BBT copolymers, they were investigated for use as donor molecules in bulk heterojunction (BHJ) OPV devices. Unfortunately, due to the very poor solubility of pBTzBBT, blend solutions with fullerene acceptors of concentrations suitable for OPV devices could not be obtained. The remaining materials were employed as donor materials, blended with [6,6]-phenyl- $\mathrm{C}_{71}$-butyric acid methyl ester $\left(\mathrm{PC}_{71} \mathrm{BM}\right)$ and fabricated into OPV devices using the conventional architecture of ITO/PEDOT:PSS/BHJ/Ca/Al unless otherwise stated. The results are summarised in Table 4.

pDPP2ThBBT and pDPPThFBBT exhibit very similar device performances across all measurements (PCEs of 0.89 and $0.87 \%$, respectively) representing a small improvement over the simpler pDPPThBBT material (PCE 0.50\%). The increased PCEs are attributed to the higher $\mathrm{FF}$ of $60 \%$ and $55 \%$ respectively, compared to $50 \%$ for pDPPThBBT. The FF obtained for each of the DPP-containing BBT copolymers increases proportionally with increasing hole mobility, which is in good agreement with previously published data. ${ }^{52}$

Table 4 OPV device data for the 4,8-BBT copolymers

\begin{tabular}{|c|c|c|c|c|c|}
\hline Polymer & $\begin{array}{l}\text { PCE } \\
\text { avg. (\%) }\end{array}$ & $\begin{array}{l}\text { PCE } \\
\text { best (\%) }\end{array}$ & $V_{\mathrm{OC}}(\mathrm{V})$ & $J_{\mathrm{SC}}\left(\mathrm{mA} \mathrm{cm}^{-2}\right)$ & $\mathrm{FF}(\%)$ \\
\hline pBTzBBT & - & & - & - & - \\
\hline pDPPThBBT & $0.5 \pm 0.03^{a}$ & $0.53^{a}$ & $0.62 \pm 0.03^{a}$ & $1.60 \pm 0.05^{a}$ & $50.2 \pm 1.0^{a}$ \\
\hline pDPPThFBBT & $0.90 \pm 0.02$ & 0.92 & $0.64 \pm 0.03$ & $2.50 \pm 0.12$ & $55.0 \pm 1.1$ \\
\hline pBT2ThBBT & $4.33 \pm 0.12$ & 4.45 & $0.65 \pm 0.02$ & $14.3 \pm 0.39$ & $48.0 \pm 1.3$ \\
\hline pBDTBBT & $0.82 \pm 0.02$ & 0.84 & $0.81 \pm 0.02$ & $2.71 \pm 0.05$ & $39 \pm 0.78$ \\
\hline
\end{tabular}

${ }^{a}$ Inverted architecture: $\mathrm{ITO} / \mathrm{Cs}_{2} \mathrm{CO}_{3} / \mathrm{BHJ} / \mathrm{MoO}_{3} / \mathrm{Ag}$. The data are for the active layer blend ratios (donor/acceptor, w/w) giving the best and average (over 4 to 8 OPV devices) power conversion efficiency, namely pDPPThBBT: PC $_{71} \mathrm{BM}, 1: 3$. pDPP2ThBBT: PC ${ }_{71} \mathrm{BM}, 1: 2$. pDPPThFBBT: $\mathrm{PC}_{71} \mathrm{BM}$, 1:3. pBT2ThBBT: PC $_{71}$ BM, 1:1 (3\% DIO). pBDTBBT: PC $_{71}$ BM, 1:1 (3\% DIO). pDTPBBT: PC $_{71}$ BM, 1:1. Optimisation details of these devices are included in the ESI. The error bars $( \pm)$ are standard deviations of the measured data. 
(a)

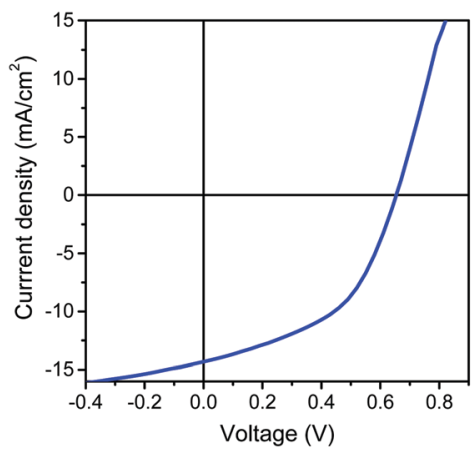

(b)

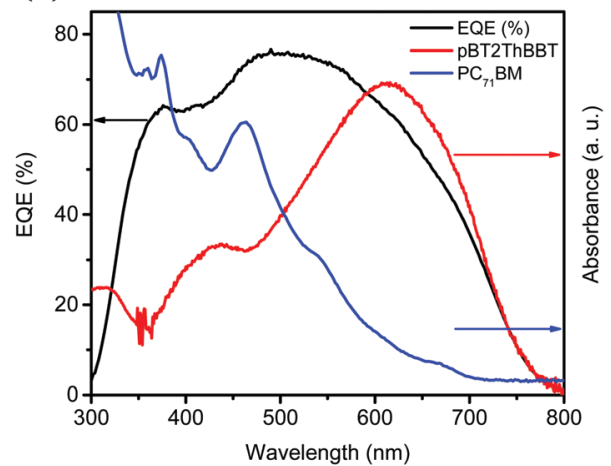

Fig. 7 (a) J-V characteristics and (b) EQE spectra for the best performing pBT2ThBBT:PC ${ }_{71} B M$ OPV devices.

The highest PCE (4.45\%) was obtained from pBT2ThBBT using a $1: 1$ blend with $\mathrm{PC}_{71} \mathrm{BM}$ and $3 \%$ diiodooctane (DIO) as an additive. The high PCE can be largely attributed to a very high $J_{\mathrm{SC}}\left(14.32 \mathrm{~mA} \mathrm{~cm}{ }^{-2}\right)$ as well as moderate $V_{\mathrm{OC}}$ and $\mathrm{FF}$ $(0.65 \mathrm{~V}, 48 \%)$. The impressive $J_{\text {sc }}$ generated from this device, comparable to that of PTB $7,^{53}$ indicates that this material is a promising candidate for high-efficiency OPV devices. The $J-V$ characteristics, external quantum efficiency (EQE) and absorption spectra of the active layer blend components (pBT2ThBBT and $\mathrm{PC}_{71} \mathrm{BM}$ ) corresponding to the best OPV device are shown in Fig. 7. The higher photocurrent of this polymer compared to others can be due to its broad absorption (as shown in Fig. 3) and the higher extinction coefficient (Fig. S44, ESI $\dagger$ ) compared to other BBT polymers. Moreover, the exciton diffusion length $\sqrt{2 D \tau}$ of pBT2ThBBT determined by time resolved fluorescence studies is found to be $\sim 10 \mathrm{~nm}$ which is higher than many donor-acceptor polymers. For example, the reported value of $\sqrt{D \tau}$ for PTB7 of $4-5 \mathrm{~nm}^{54}$ would give $\sqrt{2 D \tau}$ of 6-7 nm. This enhanced exciton diffusion length of the PBT2ThBBT polymer can also contribute towards the increased photon harvesting of the pBT2ThBBT:PC $\mathrm{PC}_{71} \mathrm{BM}$ blend. The experimental details and calculations of exciton diffusion length are included in the ESI. $\dagger$

In comparison to a 2,6-BBT analogue (PBBTzBT-DT), ${ }^{46}$ pBT2ThBBT gives a higher base PCE (4.45 vs. 2.37\%) using the same conventional device structure. However, PCEs of PBBTzBT-DT were shown to improve to $3.84 \%{ }^{46}$ and $6.53 \%{ }^{55}$ by utilising an inverted device structure and a $\mathrm{ZnO}$ electron transport layer respectively, suggesting further improvements in PBT2ThBBT based devices are possible.

\section{Summary}

Copolymers of 4,8-benzobisthiazole (BBT) with bisthienyldiketopyrrolopyrrole (DPP), dithienopyrrole (DTP), benzothiadiazole (BT), benzodithiophene (BDT) and 4,4'-dialkoxybithiazole (BTz) units have been synthesised for the first time. The resultant copolymers were found to possess optical band gaps (1.36-2.00 eV), equal to or narrower than their 2,6-BBT copolymer counterparts, with good agreement to their electrochemical band gaps
(1.26-2.24 eV). The novel materials were fabricated into BGBC OFET devices resulting in record hole mobilities amongst similar 2,6-BBT or 4,8-BBT-containing polymers (up to $2.16 \times$ $10^{-2} \mathrm{~cm}^{2} \mathrm{~V}^{-1} \mathrm{~s}^{-1}$ for pDPP2ThBBT). OFETs fabricated from pBTzBBT gave a highly optimal $I_{\text {on }} / I_{\text {off }}$ ratio $\left(10^{5}\right)$ and a low driving voltage $(-2 \mathrm{~V})$, despite poor film quality (established by AFM). Optimisation of this material/device structure is expected to result in efficient, low cost, solution-processed OFETs. OPV devices utilising the 4,8-BBT copolymers as donor materials with fullerene acceptors yielded (for the best material, pBT2ThBBT) a high short-circuit current of over $14 \mathrm{~mA} \mathrm{~cm}{ }^{-2}$ and a respectable power conversion efficiency of $4.45 \%$. Further improvements in device performance for pBT2ThBBT are expected to be realised from an inverted device structure and the use of a $\mathrm{ZnO}$ electron transport layer. ${ }^{55}$

\section{Conflicts of interest}

There are no conflicts to declare.

\section{Acknowledgements}

We are grateful to the EPSRC for funding through grants $\mathrm{EP} / \mathrm{H} 004157 / 1, \mathrm{EP} / \mathrm{L} 012294 / 1, \mathrm{EP} / \mathrm{L} 017008 / 1$ and EP/L012200/1 and to the European Research Council for funding from Grant 321305. We are grateful to the research groups of Prof. Iain McCulloch (ICL/KAUST) and Prof. Neil McKeown (Edinburgh) for generously performing GPC measurements on the polymers. PJS and IDWS are Royal Society Wolfson Research Merit Award holders. Sanjay Ghosh thanks North Maharashtra University, India, for providing study leave to do post-doctoral research at the University of St Andrews, UK. Supporting data are accessible from 10.15129/9b457e8c-12bc-4a3a-9af3-7f53474f4e5c.

\section{References}

1 J. E. Carle, M. Helgesen, M. V. Madsen, E. Bundgaard and F. C. Krebs, J. Mater. Chem. C, 2014, 2, 1290-1297.

2 Y. Hu, C. Warwick, A. Sou, L. Jiang and H. Sirringhaus, J. Mater. Chem. C, 2014, 2, 1260-1263. 
3 P. M. Beaujuge and J. M. Frechet, J. Am. Chem. Soc., 2011, 133, 20009-20029.

4 L. Torsi, M. Magliulo, K. Manoli and G. Palazzo, Chem. Soc. Rev., 2013, 42, 8612-8628.

5 G. H. Gelinck, H. E. Huitema, E. van Veenendaal, E. Cantatore, L. Schrijnemakers, J. B. van der Putten, T. C. Geuns, M. Beenhakkers, J. B. Giesbers, B. H. Huisman, E. J. Meijer, E. M. Benito, F. J. Touwslager, A. W. Marsman, B. J. van Rens and D. M. de Leeuw, Nat. Mater., 2004, 3, 106-110.

6 K. Myny, S. Steudel, P. Vicca, S. Smout, M. Beenhakkers, N. J. M. Aerle, F. Furthner, B. Putten, A. Tripathi, G. Gelinck, J. Genoe, W. Dehaene and P. Heremans, Organic RFID Tags, Springer, US, 2013.

7 Y. Zhao, Y. Guo and Y. Liu, Adv. Mater., 2013, 25, 5372-5391.

8 J. Y. Back, H. Yu, I. Song, I. Kang, H. Ahn, T. J. Shin, S.-K. Kwon, J. H. Oh and Y.-H. Kim, Chem. Mater., 2015, 27, 1732-1739.

9 H. Dong, X. Fu, J. Liu, Z. Wang and W. Hu, Adv. Mater., 2013, 25, 6158-6183.

10 G. Kim, S.-J. Kang, G. K. Dutta, Y.-K. Han, T. J. Shin, Y.-Y. Noh and C. Yang, J. Am. Chem. Soc., 2014, 136, 9477-9483.

11 B. H. Lee, G. C. Bazan and A. J. Heeger, Adv. Mater., 2016, 28, 57-62.

12 C. Luo, A. K. K. Kyaw, L. A. Perez, S. Patel, M. Wang, B. Grimm, G. C. Bazan, E. J. Kramer and A. J. Heeger, Nano Lett., 2014, 14, 2764-2771.

13 H.-R. Tseng, H. Phan, C. Luo, M. Wang, L. A. Perez, S. N. Patel, L. Ying, E. J. Kramer, T.-Q. Nguyen, G. C. Bazan and A. J. Heeger, Adv. Mater., 2014, 26, 2993-2998.

14 H. Minemawari, T. Yamada, H. Matsui, J. y. Tsutsumi, S. Haas, R. Chiba, R. Kumai and T. Hasegawa, Nature, 2011, 475, 364-367.

15 H. Sirringhaus, Adv. Mater., 2014, 26, 1319-1335.

16 C. Sinturel, M. Vayer, M. Morris and M. A. Hillmyer, Macromolecules, 2013, 46, 5399-5415.

17 G. Kim, A. R. Han, H. R. Lee, J. Lee, J. H. Oh and C. Yang, Chem. Commun., 2014, 50, 2180-2183.

18 M. Li, K. Gao, X. Wan, Q. Zhang, B. Kan, R. Xia, F. Liu, X. Yang, H. Feng, W. Ni, Y. Wang, J. Peng, H. Zhang, Z. Liang, H.-L. Yip, X. Peng, Y. Cao and Y. Chen, Nat. Photonics, 2017, 11, 85-90.

19 S. Li, L. Ye, W. Zhao, S. Zhang, S. Mukherjee, H. Ade and J. Hou, Adv. Mater., 2016, 28, 9423-9429.

20 K. Takimiya, I. Osaka and M. Nakano, Chem. Mater., 2014, 26, 587-593.

21 T. Lei, J. H. Dou, X. Y. Cao, J. Y. Wang and J. Pei, J. Am. Chem. Soc., 2013, 135, 12168-12171.

22 Y.-H. Lin, S. B. Darling, M. P. Nikiforov, J. Strzalka and R. Verduzco, Macromolecules, 2012, 45, 6571-6579.

23 K. Osowska and O. S. Miljanic, Chem. Commun., 2010, 46, 4276-4278.

24 N. E. Jackson, B. M. Savoie, K. L. Kohlstedt, M. Olvera de la Cruz, G. C. Schatz, L. X. Chen and M. A. Ratner, J. Am. Chem. Soc., 2013, 135, 10475-10483.

25 G. Conboy, H. J. Spencer, E. Angioni, A. L. Kanibolotsky, N. J. Findlay, S. J. Coles, C. Wilson, M. B. Pitak, C. Risko,
V. Coropceanu, J.-L. Bredas and P. J. Skabara, Mater. Horiz., 2016, 3, 333-339.

26 E. Zaborova, P. Chavez, R. Bechara, P. Leveque, T. Heiser, S. Mery and N. Leclerc, Chem. Commun., 2013, 49, 9938-9940. 27 R. G. D. Taylor, J. Cameron, I. A. Wright, N. Thomson, O. Avramchenko, A. L. Kanibolotsky, A. R. Inigo, T. Tuttle and P. J. Skabara, Beilstein J. Org. Chem., 2015, 11, 1148-1154.

28 S. Ando, R. Murakami, J.-i. Nishida, H. Tada, Y. Inoue, S. Tokito and Y. Yamashita, J. Am. Chem. Soc., 2005, 127, 14996-14997.

29 I. Bulut, P. Chavez, A. Mirloup, Q. Huaulme, A. Hebraud, B. Heinrich, S. Fall, S. Mery, R. Ziessel, T. Heiser, P. Leveque and N. Leclerc, J. Mater. Chem. C, 2016, 4, 4296-4303.

30 J.-L. Wang, Z. Wu, J.-S. Miao, K.-K. Liu, Z.-F. Chang, R.-B. Zhang, H.-B. Wu and Y. Cao, Chem. Mater., 2015, 27, 4338-4348.

31 J. Liu, R. Zhang, I. Osaka, S. Mishra, A. E. Javier, D.-M. Smilgies, T. Kowalewski and R. D. McCullough, Adv. Funct. Mater., 2009, 19, 3427-3434.

32 X. Guo, J. Quinn, Z. Chen, H. Usta, Y. Zheng, Y. Xia, J. W. Hennek, R. P. Ortiz, T. J. Marks and A. Facchetti, J. Am. Chem. Soc., 2013, 135, 1986-1996.

33 Y. A. Getmanenko, S. Singh, B. Sandhu, C.-Y. Wang, T. Timofeeva, B. Kippelen and S. R. Marder, J. Mater. Chem. C, 2014, 2, 124-131.

34 S.-W. Hwang, E.-R. Park, Y.-J. Chung, Y.-J. Bae and I.-T. Kim, Bull. Korean Chem. Soc., 2011, 32, 2446-2448.

35 J.-G. Kang, H.-G. Cho, S. K. Kang, C. Park, S. W. Lee, G. B. Park, J. S. Lee and I. T. Kim, J. Photochem. Photobiol., A, 2006, 183, 212-217.

36 J.-G. Kang, H.-J. Kim, Y.-K. Jeong, M.-K. Nah, C. Park, Y. J. Bae, S. W. Lee and I. T. Kim, J. Phys. Chem. B, 2010, 114, 3791-3798.

37 I. T. Kim, S. W. Lee, S. Y. Kim, J. S. Lee, G. B. Park, S. H. Lee, S. K. Kang, J.-G. Kang, C. Park and S.-H. Jin, Synth. Met., 2006, 156, 38-41.

38 Y. N. Lee, P. Attri, S. S. Kim, S. J. Lee, J. H. Kim, T. J. Cho and I. T. Kim, New J. Chem., 2017, 41, 6315-6321.

39 Y. Li, P. Sonar, L. Murphy and W. Hong, Energy Environ. Sci., 2013, 6, 1684-1710.

40 C. Liu, K. Wang, X. Gong and A. J. Heeger, Chem. Soc. Rev., 2016, 45, 4825-4846.

41 Y. Li, S. P. Singh and P. Sonar, Adv. Mater., 2010, 22, 4862-4866. 42 D. Cortizo-Lacalle, S. Arumugam, S. E. T. Elmasly, A. L. Kanibolotsky, N. J. Findlay, A. R. Inigo and P. J. Skabara, J. Mater. Chem., 2012, 22, 11310-11315.

43 L. Biniek, S. Fall, C. L. Chochos, D. V. Anokhin, D. A. Ivanov, N. Leclerc, P. Lévêque and T. Heiser, Macromolecules, 2010, 43, 9779-9786.

44 N. Wang, Z. Chen, W. Wei and Z. Jiang, J. Am. Chem. Soc., 2013, 135, 17060-17068.

45 D. E. Seitz, S.-H. Lee, R. N. Hanson and J. C. Bottaro, Synth. Commun., 1983, 13, 121-128.

46 M. Tsuji, A. Saeki, Y. Koizumi, N. Matsuyama, C. Vijayakumar and S. Seki, Adv. Funct. Mater., 2014, 24, 28-36.

47 E. Ahmed, S. Subramaniyan, F. S. Kim, H. Xin and S. A. Jenekhe, Macromolecules, 2011, 44, 7207-7219. 
48 J.-F. Chang, J. Clark, N. Zhao, H. Sirringhaus, D. W. Breiby, J. W. Andreasen, M. M. Nielsen, M. Giles, M. Heeney and I. McCulloch, Phys. Rev. B: Condens. Matter Mater. Phys., 2006, 74, 115318.

49 J. J. Intemann, K. Yao, H.-L. Yip, Y.-X. Xu, Y.-X. Li, P.-W. Liang, F.-Z. Ding, X. Li and A. K. Y. Jen, Chem. Mater., 2013, 25, 3188-3195.

50 C.-H. Kim, H. Hlaing, J.-A. Hong, J.-H. Kim, Y. Park, M. M. Payne, J. E. Anthony, Y. Bonnassieux, G. Horowitz and I. Kymissis, Adv. Mater. Interfaces, 2015, 2, 1400384.
51 P. Sonar, E. L. Williams, S. P. Singh and A. Dodabalapur, J. Mater. Chem., 2011, 21, 10532-10541.

52 M. C. Scharber, D. Mühlbacher, M. Koppe, P. Denk, C. Waldauf, A. J. Heeger and C. J. Brabec, Adv. Mater., 2006, 18, 789-794.

53 Z. He, C. Zhong, S. Su, M. Xu, H. Wu and Y. Cao, Nat. Photonics, 2012, 6, 591-595.

54 N. Zarrabi, D. M. Stoltzfus, P. L. Burn and P. E. Shaw, J. Phys. Chem. C, 2017, 121, 18412-18422.

55 A. Saeki, M. Tsuji, S. Yoshikawa, A. Gopal and S. Seki, J. Mater. Chem. A, 2014, 2, 6075-6080. 